\title{
Erratum: Inhomogeneous chiral condensate in the quark-meson model [Phys. Rev. D 96, 016013 (2017)]
}

\author{
Prabal Adhikari, Jens O. Andersen, and Patrick Kneschke
}

(Received 26 October 2018; published 12 November 2018)

DOI: 10.1103/PhysRevD.98.099902

\section{MODIFIED EQUATIONS}

Equation (6) in Ref. [1]:

$$
V_{\text {tree }}=\frac{1}{2} f_{\pi}^{2} q^{2} \frac{\Delta^{2}}{m_{q}^{2}}-\frac{1}{4} f_{\pi}^{2}\left(m_{\sigma}^{2}-3 m_{\pi}^{2}\right) \frac{\Delta^{2}}{m_{q}^{2}}+\frac{1}{8} f_{\pi}^{2}\left(m_{\sigma}^{2}-m_{\pi}^{2}\right) \frac{\Delta^{4}}{m_{q}^{4}}-m_{\pi}^{2} f_{\pi}^{2} \frac{\Delta}{m_{q}} .
$$

However, we must multiply the last term by the term $\cos (q z) \delta_{q, 0}$. The Kronecker-delta $\delta_{q, 0}$ is necessary since a term proportional to $\cos (q z)$ vanishes for nonzero $q$ upon integrating the free energy density over a sufficiently large spatial volume. The corrected equation then reads

$$
V_{\text {tree }}=\frac{1}{2} f_{\pi}^{2} q^{2} \frac{\Delta^{2}}{m_{q}^{2}}-\frac{1}{4} f_{\pi}^{2}\left(m_{\sigma}^{2}-3 m_{\pi}^{2}\right) \frac{\Delta^{2}}{m_{q}^{2}}+\frac{1}{8} f_{\pi}^{2}\left(m_{\sigma}^{2}-m_{\pi}^{2}\right) \frac{\Delta^{4}}{m_{q}^{4}}-m_{\pi}^{2} f_{\pi}^{2} \frac{\Delta}{m_{q}} \cos (q z) \delta_{q, 0} .
$$

We must multiply by the same term in Eq. (7) for the complete renormalized one-loop effective potential in the large- $N_{c}$ limit. The middle term in line four is then replaced by

$$
-m_{\pi}^{2} f_{\pi}^{2}\left[1-\frac{4 m_{q}^{2} N_{c}}{(4 \pi)^{2} f_{\pi}^{2}} m_{\pi}^{2} F^{\prime}\left(m_{\pi}^{2}\right)\right] \frac{\Delta}{m_{q}} \cos (q z) \delta_{q, 0} .
$$

The corrections, unfortunately, have consequences for our plots and conclusions regarding inhomogeneous phases at the physical point. After redoing the calculations with the correct Eq. (7) in the paper, we conclude that there are no inhomogeneous phases for $m_{\pi}=140 \mathrm{MeV}$. The plots in Fig. 5 are thus incorrect. We have redone the analysis of inhomogeneous phases as a function of the pion mass Ref. [2]. The inhomogeneous phase is disfavored for pion masses larger than $m_{\pi}=37.1 \mathrm{MeV}$. The mechanism behind this is as follows. The symmetry-breaking term $\sim-m_{\pi}^{2} f_{\pi}^{2} \cos (q z) \delta_{q, 0}$ is nonzero only for $q=0$. When the pion mass is sufficiently large, this term is large enough for the homogeneous phase to be preferred over the inhomogeneous one. We refer to Ref. [2] for a complete analysis.

[1] P. Adhikari, J. O. Andersen, and P. Kneschke, Phys. Rev. D 96, 016013 (2017).

[2] J. O. Andersen and P. Knescke, Phys. Rev. D 97, 076005 (2018). 